\title{
Update: healthcare reform - it's getting personal
}

\author{
"Measuring PDL-1 levels as a biomarker for therapy is a beginning, but we still do \\ not have a full understanding as to which patients respond best, and importantly \\ not develop autoimmune disease as T-cell function is unleashed."
}

First draft submitted: 11 April 2017; Accepted for publication: 11 May 2017; Published online: 22 June 2017

Keywords: deep machine learning $\bullet$ electronic health records $\bullet$ EHR $\bullet$ healthcare reform - 'omics' $\bullet$ personalized care $\bullet$ pharmacogenomics $\bullet$ precision medicine $\bullet$ value-based care

The difficulties encountered trying to 'repeal or replace' the US Affordable Care Act, highlight the extreme complexity of providing affordable care for all while not bankrupting the nation. High-value healthcare (quality/cost) can only be achieved by physicians (working with their health systems and insurers) and scientists. The role of the government should be to: align incentives for healthcare providers to develop new models of value-based personalized care, and support the revolution in genomic and computational sciences that can bring precision medicine to the mainstream.

5 years ago this author had written about the potential of personalized medicine to improve healthcare in this journal [1]. Since that time, there has been steady but incremental progress. New models of clinical care emphasize the individual patient at the center of team care. Patient's goals are being increasingly heard and doctors are becoming less shy in asking the simple yet critical question: what does the patient want? There has also been progress in using one's genotype to define phenotype, particularly with respect to oncology and pharmacogenomics.

Last year, investigators used expression profiling to identify a subset of thyroid tumors with such a benign course that a consensus group redefined the lesion as "noninvasive follicular thyroid neoplasm with papillary-like nuclear features" [2]. The name does not exactly roll off the tongue, but it represents the first time we have reclassified a cancer and called it benign. The hottest topic in cancer is immunotherapy, where responses are being observed to advanced tumors previously unimaginable. For example, targeting checkpoint inhibitors, such as PDL-1, unlocks $T$ cells allowing them to act on a variety of tumors [3]. Measuring PDL-1 levels as a biomarker for therapy is a beginning, but we still do not have a full understanding as to which patients respond best, and importantly not develop autoimmune disease as T-cell function is unleashed. Further understanding of additional checkpoint inhibitors, the complicated biology within the tumor microenvironment and tumor antigen repertoires will guide future treatment.

Pharmacogenomics, the study of genomic variations that influence individual response to drugs, is well poised to enter mainstream care since we already know drug disposition pathways and many of the polymorphisms that alter drug metabolism [4]. One test beginning to make its way into routine clinical use is testing for polymorphisms of the CYP450 isozyme 2C19i, which is responsible for hepatic activation of the prodrug clopidogrel allowing it to inhibit platelets. Following cardiac stenting, clopidogrel is used to prevent clots, hence it is imperative to

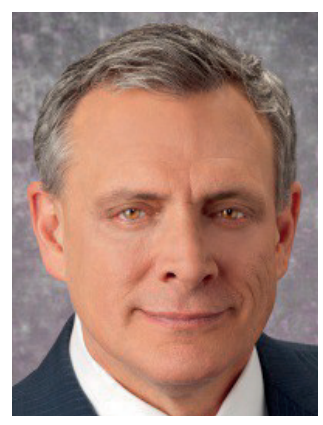

Steven D Shapiro Author for correspondence: Department of Medicine, University of Pittsburgh School of Medicine \& UPMC, Pittsburgh, PA 15261, USA Tel.: +1 4126053995 shapirosd@upmc.edu 
know which patients will and will not benefit [5]. As we determine how much clinical data are needed to adopt tests clinically, and how to accommodate pharmacogenomics in the electronic health record (EHR), many new tests will become routine.

Despite long queuing for EHR modifications, the fact that medical records are almost entirely electronic provides the data to match genotype with phenotype [6]. In the DiscovEHR study [7], 50,726 exomes were sequenced identifying approximately 4.2 million rare single-nucleotide variants and insertion/deletion events. About 3.5\% of individuals harbored deleterious variants in 76 clinically actionable genes. This is particularly striking given the fact that most of the interesting single-nucleotide polymorphisms (SNPs) from wholegenome association studies are located in the noncoding introns that compose the vast majority of our genome. As the price of whole-genome sequencing continues to decrease and our ability to understand the functional implications of intronic variants increases, application of genetic medicine will take a central role.

In addition to the 'phenome' and genome, there has been progress in a variety of other 'omics' including the transcriptome, proteome and a variety of protein processing pathways such as the metabolome, epigenome and immunogenome. Outnumbering host cells, the microbiome has also been shown to be important in shaping health and predisposing to disease. Our ability to probe expression in single cells and tissues will further aid our understanding of complex tissue and tumor biology as well as spatial diversity.

Hence, we are now poised to go beyond genotype-phenotype correlation. Large datasets composed of multiple types of 'omic' information may be mined and applied to deep machine learning. This process will generate algorithms that learn from and make predictions about the data using multiple levels of representation and abstraction, resulting in a comprehensive view of one's health status over time. In other words, we can give clinicians a 'crystal ball' displaying all of the health problems a patient is at risk to encounter over time, and for the diseases that the patient has, to visualize the future course of that disease including severity and progression, complications and comorbidities. Moreover, ideal treatment plans could be developed taking into account efficacy and cost, in the context of the patient's goals. This will be precision medicine enabled personalized care.

Longitudinal multiomics profiling remains a future goal as we do not have the math developed or computational power to handle multiple 'omics' and the artificial intelligence to produce the 'crystal ball'. Yet, Moore's law gives optimism that the incredible pace of progress will continue. In the late 1960s, Thomas Moore observed that the number of transistors on a microprocessor chip will double every year or so with corresponding increase in chip performance, computational speed and power. This has now slowed down to doubling every $2-2.5$ years, and we are reaching the limits of size reduction (currently chips are $10 \mathrm{nM}$ with theoretical limit of $\sim 2 \mathrm{nM}$ ). New computing methods must be developed, and there are many candidates ranging from quantum computing, 2D graphene-like compounds, to spintronic materials [8]. Increased artificial intelligence itself will contribute to the development of new methods to maintain the pace of progress. As a result we should continue this incredible increase in our ability to analyze larger and more complex datasets that are acquired in an asynchronous temporal manner resulting in improved human health.

One must be careful not to oversell the promise of science and technology. In fact, science and technology is in part responsible for the current state of our inflated healthcare system. Breakthroughs have led to significant progress treating acute, life-threatening illness and have turned some severe acute illnesses to chronic condition. Despite few cures, we have modified disease course resulting in more patients living longer with multiple chronic ailments. This certainly benefits the individual (as long as they do not suffer), but society bears an unsustainable financial burden. The next wave of scientific and technological advances through precision medicine powered by artificial intelligence must advance value-based care and hopefully understand and cure chronic diseases.

To promote value-based care, we must not only identify those patients who will not benefit from expensive, aggressive therapy but have the will as a society to limit care for those where the expenses are below a certain dollar spent per life year gained $(\$ 40,000-\$ 50,000$ generally accepted). To begin, we should continue to discuss the wishes and goals of each patient (and what they can afford in the world of high-deductible/coinsurance world). To cure disease, once we have used deep machine learning to identify disease course, we must apply the emerging science of causal modeling to develop 'in silico' molecular disease networks. This will give investigators hypotheses to be tested and pathways to intervene leading to cure.

Finally, the greatest objection to the American Healthcare Act replacement of the Affordable Care Act was its abandonment of the poor. Improving the human condition has always had the greatest impact on a population's health, far more than medical care. Government grants for insurance companies to address long-term support services focusing on adequate food, shelter and safety is perhaps the most important and least known contribution of health reform [9]. This will turn hopelessness and the opioid epidemic to self-worth and a productive society eager to learn new skills in this Moore's law-induced rapidly changing 
world. Helping every person fulfill their ambitions is personalized medicine in a population health setting at its best.

\section{Financial \& competing interests disclosure}

The author has no relevant affiliations or financial involvement with any organization or entity with a financial interest in or financial conflict with the subject matter or materials discussed in the manuscript. This includes employment, consultancies, honoraria, stock ownership or options, expert testimony, grants or patents received or pending, or royalties.

No writing assistance was utilized in the production of this manuscript.

\section{References}

1 Shapiro SD. Healthcare reform: it's getting personal. Personal. Med. 9, 405-412 (2012).

2 Nikiforov YE, Seethala RR, Tallini G et al. Nomenclature revision for encapsulated follicular variant of papillary thyroid carcinoma: a paradigm shift to reduce overtreatment of indolent tumors. JAMA Oncol. 2(8), 1023-1029 (2016).

3 Topalian SL, Taube JM, Anders RA, Pardoli DM. Mechanismdriven biomarkers to guide immune checkpoint blockade in cancer therapy. Nat. Rev. Cancer 16(5), 275-287 (2016). Empey PE. Pharmacogenomics to achieve precision medicine. Am. J. Health Syst. Pharm. 73(23), 1096-1097 (2016).

5 Cavallari LH, Weitzel KW, Elsey AR, Liu X, Mosley SA, Smith DM et al. Institutional profile: University

of Florida health personalized medicine program. Pharmacogenomics 18(5), 421-426 (2017).

6 Wu P-Y, Cheng C-W, Chanchala DK, Venugopalan J, Hoffman R, Wang MD. -Omic and electronic health record big data analytics for precision medicine. IEEE Trans. Biomed. Eng. 64, 263-271 (2017).

7 Dewey FE, Murray MF, Overton JD et al. Distribution and clinical impact of functional variants in 50,726 whole-exome sequences from the DiscovEHR study. Science 354 (6319), pii:aaf6814 (2016).

8 Waldrop MM. More than Moore. Nature 530, 144-146 (2016).

9 Medicaid. Long term services and supports. www.medicaid.gov/medicaid/ltss 